\title{
Entrevista a Gui Bonsiepe
}

En 1968 el diseñador alemán Gui Bonsiepe inicia su trabajo en Chile, provocando con ello un cambio determinante en el discurso y práctica del diseño nacional al incorporar en la disciplina un necesario "sentido proyectual". Asimismo, forma la sección de Diseño Industrial del Instituto de Investigación Tecnológica de Corfo, que permanece hasta 1973. A treinta años de su trabajo en Chile, Bonsiepe elabora una propuesta reinterpretativa del diseño, trasladando al proyecto desde el racionalismo al lenguaje. Palmarola aborda, a través de una entrevista, los distintos aspectos de esta propuesta de diseño.

In 1968 the German designer Gui Bonsiepe began bis work in Chile, causing a decisive change in the speech and practice of Chilean design, incorporating in the discipline a necessary "project sense". Also, he formed the section of Industrial Design of the Institute of Technological Investigation of CORFO that remains up to 1973. After thirty years of his work in Chile, Bonsiepe elaborates a new proposal for design, transferring the project from rationalism to a problem of language. Palmarola approaches, through an interview, the different aspects of this design proposal.

\section{El Proyecto en Diseño}

Con el cierre de la Hochschule für Gestaltung de Ulm en 1968, y con la diáspora de sus profesores, el modelo metodológico proyectual se extiende a los más diversos países, influyendo considerablemente con un carácter racionalista y crítico la condición del diseño. Ese mismo año, en el marco de un programa de las Naciones Unidas para el desarrollo de la pequeña y mediana industria, el diseñador (HfG) alemán Gui Bonsiepe inicia su trabajo en Chile, inaugurando así un cambio determinante en el discurso y práctica del diseño nacional al incorporar en la disciplina un necesario "sentido proyectual".

El lenguaje técnico y pragmático del discurso "ulmiano" permitiría en gran medida la integración del diseño en las industrias e instituciones estatales chilenas. En este contexto, es creada en 1971 la nueva Sección de Diseño Industrial del Instituto de Investigaciones Tecnológicas (INTEC) de CORFO. Bajo la dirección de Bonsiepe, este grupo inicia el diseño de una gran cantidad de productos para ser fabricados industrialmente. El objetivo principal de este proyecto demandado por el gobierno socialista concentraba los esfuerzos de diseño en la solución de problemas sociales masivos, la reducción de la dependencia tecnológica y el desarrollo de la industria nacional. La variedad de productos comprendía dosificadores para la leche en polvo, vajilla de loza, mobiliario para viviendas básicas, tocadiscos portátiles, calculadoras, maquinaria agrícola, entre otros, conformando así el primer grupo de productos diseñados en el país y fabricados masivamente. Aunque Bonsiepe nunca formaría parte de un plantel docente en Chile, con sus propuestas motivó una reformulación de los contenidos curriculares al interior de las principales escuelas de diseño, vinculando el ejercicio académico al quehacer productivo.

Se inicia así, mediante una incorporación concreta del diseño en la industria y en la sociedad, la fase de gestación e institucionalización del diseño, consolidándose en el país un nuevo discurso de lo que habría sido hasta entonces una indefinida y difusa actividad.

Tras el 11 de septiembre de 1973, con la caída del gobierno de Salvador Allende, los procesos de diseño liderados por Bonsiepe y su grupo desaparecen. Las experiencias en torno a estos proyectos serían luego presentadas en su libro Teoría y Práctica del Diseño Industrial publicado en Italia el año 1975.

\section{Interfase y lenguaje}

A treinta años del trabajo de Bonsiepe en Chile, fenómenos como la globalización y la informática transforman radicalmente nuestra forma de pensar sobre el mundo y el diseño. Bajo este contexto Bonsiepe, luego de trabajar en Argentina y Brasil, se traslada en 1987 a Berkeley, California, donde se especializa en el diseño de "buman user interfaces" en una empresa de software, comenzando así una segunda etapa en su discurso de diseño.

Con el desarrollo de la informática, nuevas relaciones sobre los actos de uso, el conocimiento y el aprendizaje hacían evidente el carácter inmaterial del diseño al momento de procurar en un software su disponibilidad y efectividad en las acciones que anticipaba. Así, el concepto de "interfase" cobra fuerza y la materialidad específica del objeto con la que hasta entonces se había vinculado al diseño comienza a desaparecer.

Por otra parte, en Berkeley nuevos modos de pensar y trabajar sobre el lenguaje, basados en aportes de actores tan diversos como Martin Heidegger y Humberto Maturana, generaban cambios radicales en distintas áreas, abriendo también nuevos modos de mirar y actuar en el diseño. Una interpretación de los seres humanos como seres lingüísticos, que se crean a sí mismos en el lenguaje y a través de él, modificaba una larga tradición sobre el concepto de lenguaje. Lo que habría sido hasta entonces sólo un instrumento descriptivo de una realidad interna o externa, es entendido ahora como un dominio "generativo" capaz de crear nuevas realidades. A partir de estas experiencias, Bonsiepe elabora una propuesta reinterpretativa del diseño mante- niendo invariable su carácter crítico y moderno. El proyecto desde el racionalismo se traslada ahora a un proyecto desde el lenguaje.

En su esencia esta propuesta contempla un concepto amplio de interfase, sobre la cual se especificaría todo campo de acción del proyecto de diseño. Usuario, artefacto y acción se conectan a partir de este espacio, donde la preocupación de su diseño se concentra en la disponibilidad del producto y sus acciones eficientes, revelando así una situación común a todas las categorías de diseño.

Dicha reinterpretación plantea además un origen y fundamento del diseño a partir de las distinciones que un observador trae a la mano en el lenguaje, siendo sólo sobre estas distinciones creadas y diseñadas sus funciones. De esta manera, una forma inicialmente nada tiene que ver con las funciones, éstas no se encuentran en los productos, sino en el lenguaje y es dentro de éste donde se articula el espacio de distinciones que hacen disponible a un objeto. Esta interpretación sobre el lenguaje, asimilada en la propuesta de Bonsiepe, otorgaría al diseño un papel predominantemente activo de participación sobre las posibilidades y modos de ser en el mundo, ya que como reorganización del espacio de distinciones, crea un nuevo lenguaje y un nuevo modo de ser.

$\mathrm{Su}$ último libro Dall'oggetto allinterfaccia, Feltrinelli, Milán, 1995 (versión castellana Del objeto a la interfase, Ediciones Infinito, Buenos Aires, 1999) da cuenta de este radical cambio conceptual. Para profundizar sobre las principales tesis de esta propuesta de diseño conversamos con Gui Bonsiepe ${ }^{1}$.

¿A qué necesidad responde su propuesta reinterpretativa del diseño?

No a una necesidad específica, pero sí a mi disconformidad con los tópicos y esquemas interpretativos con su vocabulario recurrente de forma, función, racionalismo, funcionalismo, design brief, semántica del producto, emoción versus racionalidad. Busqué una base a partir de la cual se puede referir al diseño tanto de artefactos físicos (diseño industrial) como al diseño de artefactos comunicativos (diseño gráfico y comunicación visual). Me dejaron insatisfecho las contribuciones postmodernistas y los intentos de estudios de diseño que se expandieron en el discurso de diseño de manera puramente literaria, sin base empírica, erigiendo un mundo cerrado, académico, en el cual los teóricos hablan solamente a otros teóricos -un revival de un nuevo escolasticismo ensimismado y de un preciosismo definitorio-. De ahí pueden surgir 


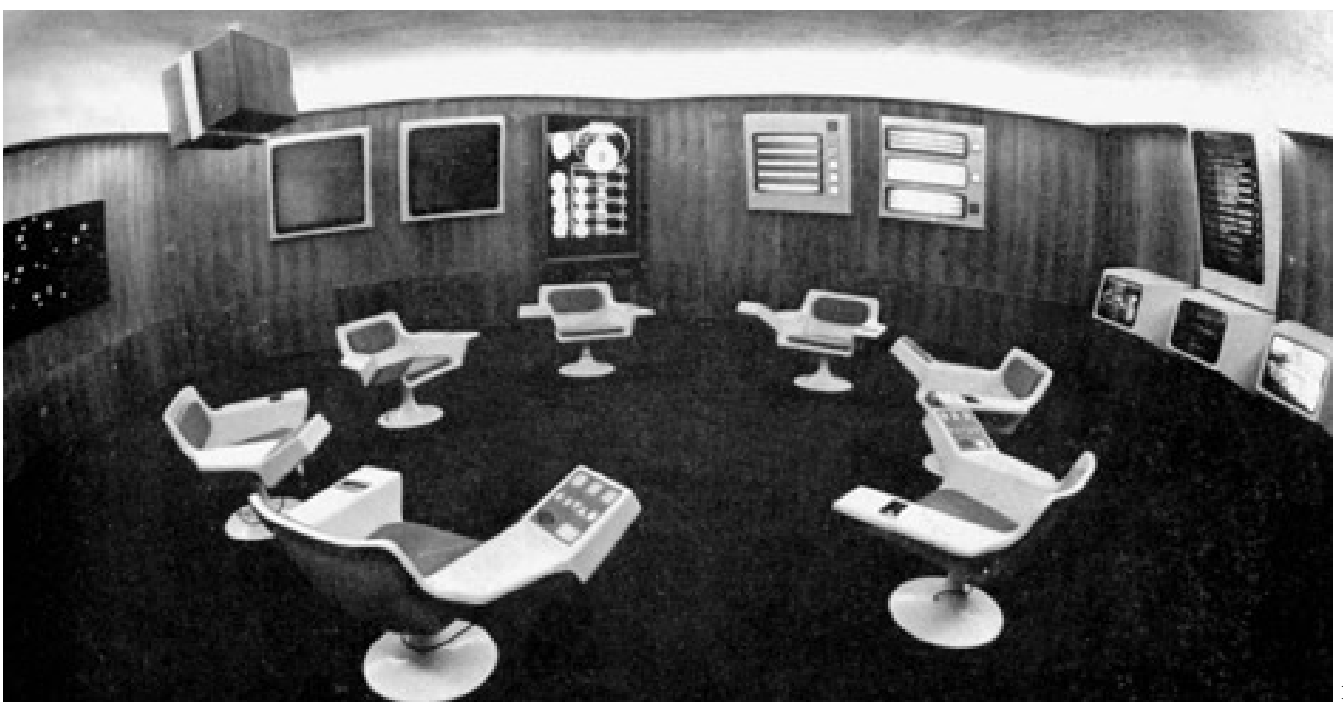

las más raras reflexiones sobre la realidad del diseño. Más extraña aún resulta cuando cultivan pretensiones normativas sobre todo en el campo de la enseñanza e investigación del diseño. No se crea una teoría del diseño quedándose solamente en el espacio discursivo, leyendo textos -aunque esto es imprescindible y recomendar una mayor práctica de lectura no hará mal a los estudiantes del diseño-.

Desde Heidegger en filosofía, a Maturana en la biología, el lenguaje ya no es visto como un medio operativo sino como un medio constitutivo. ¿Que implicancias tendrían estas ideas sobre los conceptos tradicionales de función e identidad en diseño?

El lenguaje "revela" y no reproduce una realidad. Análogamente el acto proyectual constituye un espacio de acción. Por eso el diseño deriva de nada, sobre todo no de las funciones supuestamente objetivas. El diseño constituye funciones, no las satisface. También las funciones son diseñadas.

El nudo del diseño, la médula de la identidad del diseño, consiste -en esto similar al lenguajeen constituir una realidad. Diseño y lenguaje son dos dimensiones constitutivas antropológicas.

En el discurso general de diseño, ¿dónde cree Ud. que nace el interés por incorporar teorías sobre lenguaje? ¿Nace en el diseño posmoderno?

No, el diseño posmoderno es en buena parte una mezcla de los mas variados ingredientes programáticos. Sirvió entre otros para instalar el discurso neoconservador en arquitectura y diseño, claro, sin jamás admitir esto, pues como el prefijo post sugiere, se pretende haber superado el modernismo del cual en general se hace una presentación caricaturesca.

¿A qué se refiere Ud. al plantear el concepto de "disponibilidad" (Zuhandenheit) en Heidegger, como una categoría central del diseño?

Heidegger distingue entre disponibilidad "Zuhandenheit" (ready-to-hand) y "Vorhandenheit" (presentat-hand) en el sentido de estar ahí físicamente. Un simple ejemplo puede ilustrar la diferencia entre estos dos conceptos: caminando por la playa se encuentra una piedra -ella está ahí, está presente-. Pero solamente mediante un acto de transformación esta piedra se revela como una posible herramienta, en algo disponible para un uso. Pasa del dominio de la mera presencia física, de lo dado, a la presencia para alguien. Ahí entra en escena el concepto del interés, más allá de la mera necesidad (need). Por medio de actos proyectuales la materialidad bruta se transforma en artefacto ligado a un interés.

¿Qué efectos considera ha tenido el trabajo de Fernando Flores en el desarrollo del diseño? ¿Cuál ha sido su experiencia al respecto?

Hace 15 años Fernando Flores junto con Terry Winograd formularon un nuevo approach al diseño basado en el lenguaje. Pero temo que su aporte no ha penetrado profundamente en el discurso de los profesionales del diseño y el cuerpo docente -al final, coffee table books llenos de bonitas ilustraciones son mas fáciles de asimilar que libros con reflexiones sobre el diseño-. Ellos han hecho un trabajo similar a la contribución de

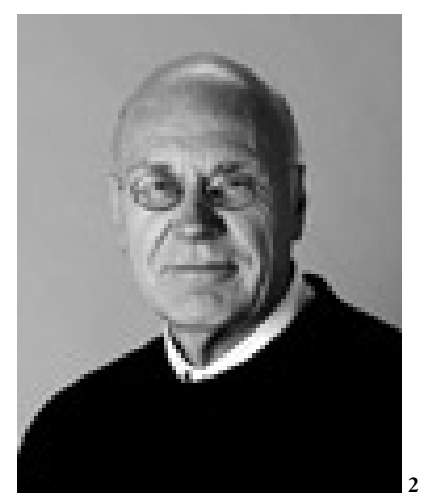

Herbert Simon a fines de la década de los ' 60 con su libro Sciences of the Artificial.

Después de la devastadora crítica de Adorno a la yerga de la autenticidad (Fargon der Eigentlichkeit) Heidegger me pareció acabado y no creía que iba a volver a leer sus textos. Pero en el ambiente de Berkeley con un intérprete clarificador como Dreyfus me acerqué de nuevo a los escritos de Heidegger y algunos de sus conceptos me parecieron útiles para esclarecer ciertos aspectos del diseño -aunque Heidegger mismo no ha desarrollado una teoría del proyecto, tal vez por su postura distanciada a la tecnología-.

¿En qué medida el carácter inmaterial de la informática ha contribuido a clarificar y expandir el concepto de "interfase" a otras categorías de diseño como el diseño industrial? El diseño encuentra su identidad en hacer disponible artefactos materiales e inmateriales. Este espacio en el cual se estructura la interacción entre artefacto y usuario lo llamo "interfase", pues este concepto contiene un potencial interpretativo más rico que el viejo binomio forma-función. Diseño de producto desde la perspectiva del diseñador industrial significa concentrarse en la interfase, su calidad de uso y calidad estéticoformal, sin descuidar obviamente otros factores como costos, producción, viabilidad económica. La esencia de la contribución del diseñador industrial a la conformación de productos consiste en estructurar la interfase y en este aspecto su enfoque difiere del trabajo de las ingenierías.

Sobre este concepto, ¿qué relevancia como referente otorga al proyecto para el "Centro 
3 "El esquema está compuesto por tres ámbitos unidos por una categoría central. En primer lugar existe un usuario o un agente social, que desea fective social, que desea efectivannente cumplir una accón. En segun él mismo quiere ejecutar (...) E tercer lugar existe un utensilio o un artefacto del que necesita el agente para llevar a término la acción (...) La conexión entre estos tres campos se produce través de la interfase (...) La interfase es el ámbito centra hacia el que se orienta el interés del diseñador. Gracias a la del disena. Gracias a la proyección de la interfase se articula cla de acción la etapa de utilización de los productos; la interfase vuelve accesible el carácter instrument de los objetos y el contenido comunicativo de la información.
Transforma los objetos en productos; transforma la simpl existencia física (Vorhandenheit) en el sentido de Heidegrer, en disponibilidad (Zuhandenheit). disponibilidad (Zuhanden S1 logramos entender con precision las connotaciones de exte ámbito constitutivo, sin el cual no existirían los utensilios, se podrá dar al diseño industrial un legitimación material mucho más firme e inobjetable con relación a interpretaciones que quieren orientarlo exclusivamente hacia la dimensión formal y estética". (Originalmente publicado e "Informe para el Encuentro Cultur Informe para el Encuentro Cultur y Nuevos Conocimientos" de Gui Bonsiepe. Universidad Autónom Metropolitana (UAM), Unidad Azcapotzalco, México, 17-20 de febrero de 1992. Publicado en Dall'oggetto all'interfaccia, Feltrinelli, Milán, 1995). de Planificación Cibernética" que Ud. dirigió en Chile el año 1972?

En 1972 no teníamos el término "interfase", tal vez ni existía en el campo de la informática. Pero visto desde hoy podemos decir que diseñábamos una interfase para poder intervenir en algunas variables neurálgicas de la economía, sus centros de producción y de distribución. Hoy tenemos otro concepto para el trabajo hecho hace 30 años: visualización de datos complejos para hacerlos comprensibles. Ahí tocamos una de las áreas más estimulantes del diseñador actual: usar el poder del espacio retínico (retinal space) para fines cognoscitivos y operativos.

Parece un cuento de ciencia ficción, pero es un hecho que en aquel momento en Chile se trabajaba en un frente de vanguardia temática. Lo más llamativo quizás es el hecho de que este trabajo había sido demandado por un ente público -tan denigrado y hasta diabolizado por los cantantes de las virtudes celestiales del mercado-.

Con el avance de la informática y las tecnologías de visualización proporcionada por ella este campo se ha expandido enormemente. Pero me pregunto: ¿la enseñanza de diseño ha acompañado este proceso? ¿o nos ha relegado al rincón de los pixel movers que sólo manejan Photoshop para fines estetizantes?

La saturación en "diseño gráfico" ha obligado a desplazar este término hacia uno de "diseño de información", priorizando una estructuración eficaz. ¿Qué paralelo conceptual se puede establecer al interior del término "diseño industrial"?

El término "diseño gráfico" hoy en día me parece haber perdido vigencia. Pues pone énfasis en el medio, es decir, los recursos gráficos y no al objeto del diseño que es la información. En el "Manifiesto Oullim" de Seoul, en cuya formulación participé como miembro de uno de los dos grupos de trabajo el año pasado, se reconoció la pérdida de vigencia del término diseño gráfico. Obviamente no se trata de cambiar un término por otro, sino de reflexionar sobre las consecuencias sobre todo para la enseñanza del diseño. $Y$ éstas pueden ser dramáticas.

El término "diseño industrial" no me parece estar afectado por este proceso de desgaste.

En la actualidad existe una propuesta de diseño orientada a dar respuesta a hábitos muy específicos de pequeños grupos. Ésta sostiene que sólo las pequeñas y medianas empresas estarían preparadas para responder a este cambio en las demandas hacia pequeñas

\section{esquema ontológico del diseño}



soluciones, no así industrias de producción a gran escala (ponencia chilena "ICSID 2001 Seoul”) ¿Cuál es su opinión al respecto?

Esta afirmación puede solamente confirmarse mediante estudios concretos. Pero podemos observar que la dicotomía entre producción a pequeña escala (hecha en la pequeña industria) y gran serie (hecha en la gran industria) ha perdido hoy su base empírica. Pues es en el campo de la más avanzada tecnología de producción que se puede registrar una producción personalizada, como ya ocurre en algunas industrias automotrices.

En relación a esto, le hago extensiva una pregunta que Alex Kufus realiza en el texto para la exposición alemana "conscientesencillo": "¿Podría haber un estilo, no desde el punto de vista del consumidor si no del fabricante, orientado a la producción de relaciones razonables y sustentables?"

Un fabricante, un manager puede implementar una política de empresa incorporando factores ambientales y estableciendo una relación duradera entre cliente y empresa. Esto define el CI de la empresa e implica resistir al cambio casi histérico de un modelo por otro modelo. Implica long-life design.

\section{2 de Julio del 2001. Colonia, Alemania}

En la actualidad Gui Bonsiepe desarrolla su trabajo como docente e investigador en la Facultad de Diseño de la Universidad de Ciencias Aplicadas en Colonia, Alemania, además de ser coordinador del Master en Diseño de Información en la Universidad de las Américas, Puebla, México, y consultor de empresas en Brasil.
Hugo Palmarola Sagredo Pontificia Universidad Católica de Chile. Estudiante del Instituto de Formación Matríztica. Ha realizado estudios de teoría e historia del arte en la Universidad Nacional Autónoma de México (UNAM), y participado como expositor de diseño en Napier University, Escocia. Actualmente es sobre referentes del diseño industrial en Chile bajo la coordinación de Alberto González.
Estudiante de la Escuela de Diseño y del Instituto de Estética de la ayudante en la Escuela de Diseño de la PUC y realiza una investigación 